\title{
Working Memory Capacity Predicts Effects of Methylphenidate on Reversal Learning
}

\author{
Marieke E van der Schaaf*, , Sean J Fallon ${ }^{2}$, Niels ter Huurne', Jan Buitelaar, ${ }^{3,4}$ and Roshan Cools ${ }^{1,2}$ \\ 'Department of Psychiatry, Radboud University Nijmegen Medical Centre, Nijmegen, The Netherlands; ${ }^{2}$ Centre for Cognitive Neuroimaging, \\ Donders Institute for Brain, Cognition and Behaviour, Radboud University Nijmegen, Nijmegen, The Netherlands; ${ }^{3}$ Department of Cognitive \\ Neuroscience, Donders Institute for Brain, Cognition and Behaviour, Radboud University Nijmegen Medical Centre, Nijmegen, The Netherlands; \\ ${ }^{4}$ Karakter Child and Adolescent Psychiatry University Center, Nijmegen, The Netherlands
}

\begin{abstract}
Increased use of stimulant medication, such as methylphenidate, by healthy college students has raised questions about its cognitiveenhancing effects. Methylphenidate acts by increasing extracellular catecholamine levels and is generally accepted to remediate cognitive and reward deficits in patients with attention deficit hyperactivity disorder. However, the cognitive-enhancing effects of such 'smart drugs' in the healthy population are still unclear. Here, we investigated effects of methylphenidate (Ritalin, $20 \mathrm{mg}$ ) on reward and punishment learning in healthy students $(N=19)$ in a within-subject, double-blind, placebo-controlled cross-over design. Results revealed that methylphenidate effects varied both as a function of task demands and as a function of baseline working memory capacity. Specifically, methylphenidate improved reward vs punishment learning in high-working memory subjects, whereas it impaired reward vs punishment learning in low-working memory subjects. These results contribute to our understanding of individual differences in the cognitiveenhancing effects of methylphenidate in the healthy population. Moreover, they highlight the importance of taking into account both inter- and intra-individual differences in dopaminergic drug research.

Neuropsychopharmacology (2013) 38, 20 I I-20 I 8; doi: I 0.1038/npp.2013.100; published online 8 May 20I3
\end{abstract}

Keywords: cognition; cognitive enhancement; dopamine; learning \& memory; psychostimulants; punishment; reversal learning; reward; Ritalin

\section{INTRODUCTION}

Psychostimulants such as methylphenidate (MPH) are the first-line medication treatment of attention deficit hyperactivity disorder (ADHD). In the clinical context, MPH decreases symptoms of inattention and hyperactivity (Faraone and Buitelaar, 2010) and may improve attentional and academic performance (Marcus and Durkin, 2011; Wigal et al, 2011). However, recent surveys report that healthy college students also increasingly use these stimulants to enhance concentration and study performance (Bundt et al, 2012; Smith and Farah, 2011). This raises questions about their cognitive-enhancing effects in the healthy population (Greely et al, 2008). MPH primarily acts by blocking the dopamine transporter (DAT), which removes excessive dopamine from the synaptic cleft, resulting in the increase of extracellular dopamine levels (Volkow et al, 2002). In ADHD, MPH is thought to be effective by restoring deficient catecholamine levels. How-

\footnotetext{
*Correspondence: Dr ME van der Schaaf, Donders Centre for Cognitive Neuroimaging, PO Box 9101, 6500 HB Nijmegen, The Netherlands. Tel: +3| 24366 8292, Fax: +31 2436 | 0989,

E-mail: Marieke.vanderschaaf@donders.ru.nl

Received 21 December 2012; revised 5 April 2013; accepted 8 April 2013; accepted article preview online 23 April 2013
}

ever, effects are still unclear in the healthy population, in which catecholamine levels are generally assumed to be relatively optimized in comparison to individuals with disorders that implicate dopamine.

There is a large variability in the cognitive-enhancing effects of psychostimulants in healthy adults (Smith and Farah, 2011) and there are numerous factors that may contribute to this variability. First, dopaminergic drug effects can vary as a function of task demands, so that some tasks are improved while other tasks are impaired (Cools and D'Esposito, 2011). For example, administration of dopamine receptor agonists improves learning from reward while impairing learning from punishment in the same subjects (Bodi et al, 2009; Cools et al, 2009; Frank and O'Reilly, 2006). This concurs with observations that psychostimulant treatment such as methylphenidate improves reward but not punishment learning in patients with ADHD (Frank et al, 2007). Such differential effects likely reflect distinct optimal levels of striatal dopamine, with high dopamine levels facilitating learning from reward and low dopamine levels facilitating learning from punishment (Frank, 2005). Here we assessed whether methylphenidate also has opposite effects on reward and punishment learning in healthy adults, thus extending methylphenidate's therapeutic effects in ADHD (Frank et al, 2007) to effects of so-called cognitive enhancers in the 
healthy population. A second factor is the large interindividual variability in drug response (Cools and D'Esposito, 2011). The same drug and doses can have different, even opposite, effects between different individuals depending on clinical condition (Mehta et al, 2004; Voon et al, 2010), baseline working memory capacity (Frank and O'Reilly, 2006; Kimberg et al, 1997; Mehta et al, 2000; Mehta et al, 2001; van der Schaaf et al, 2012), and baseline levels of dopamine function as measured with positron emission tomography (PET) (Cools et al, 2009). For example, bromocriptine was shown to improve reward learning in subjects with low baseline levels of dopamine synthesis capacity, whereas impairing it in subjects with high baseline levels of dopamine synthesis capacity (Cools et al, 2009). Accordingly, it has been suggested that the effects of dopamine receptor agents depend on the baseline state of the system (Cools and D'Esposito, 2011). By analogy, effects of MPH might also depend on the baseline state of the system.

To test these hypotheses, we administered a single dose of methylphenidate and placebo to healthy controls in a double-blind, placebo-controlled cross-over design and we stratified our drug effects by baseline working memory capacity; a measure that has previously been shown to be positively associated with striatal dopamine synthesis capacity (Cools et al, 2008; Landau et al, 2009). The direction of the relationship between MPH effects and baseline working memory capacity might parallel that seen for dopamine receptor agonists (Cools et al, 2009; Frank and O'Reilly, 2006; Mehta et al, 2000, 2001), with greater improvements in reward $v s$ punishment learning in lowthan high-working memory subjects. Alternatively, it has been proposed that DAT blockade leads to larger increases in extracellular dopamine in subjects with a higher rate of dopamine release (Volkow et al, 2002). This is supported by animal work showing baseline-dependent effects of DAT blockade on extracellular dopamine concentrations (Hooks et al, 1992). Accordingly, together with the literature reviewed above, an alternative hypothesis would be that $\mathrm{MPH}$ would induce larger improvements on reward vs punishment learning in subjects with high-working memory capacity.

\section{MATERIALS AND METHODS}

\section{Subjects}

Twenty-four subjects, recruited via campus advertisements, gave written informed consent approved by the local research ethics committee ('Commissie Mensgebonden Onderzoek', Arnhem-Nijmegen, number 2010/283) and were compensated for participation. The current task was part of a larger protocol and was preceded by a functional magnetic resonance imaging (fMRI) experiment and followed by two behavioral experiments (reported elsewhere). Five non-native Dutch speakers (ie, German) were excluded from analysis because good practice of the Dutch language was essential for optimal assessment of baseline working memory capacity as measured with the listening span and digit span. The remaining 19 subjects (mean age: 20.9 years, range: $19.0-24.4)$ were healthy, right-handed men (9) and women (10) with no relevant medical/ psychiatric history or a history of drug abuse and/or dependence. Other exclusion criteria included fulfillment of ADHD criteria, family history of schizophrenia, bipolar disorder, depression or neurological abnormalities, alcohol use of more than 20 units per week, habitual smoking ( $>20$ cigarettes per week), use of prescribed or over-the-counter medication within the last month (with the exception of occasional paracetamol and anti-conceptive medication), use of recreational drugs within 2 weeks before testing, and a history of frequent use of recreational drugs or psychotropic medication (cannabis: more than biweekly on average or periods using more than weekly; other recreational drugs (eg cocaine, amphetamines): more than five times ever; psychotropic medication: more than biweekly or periods of using more than five times weekly).

\section{Intake Procedure}

During a first intake, all subjects were screened by a medical doctor (NtH) and psychologist (MvdS), which included physical examination of weight, pulse rate, and blood pressure, medical examination and administration of the Mini-International Neuropsychiatric Interview (M.I.N.I.) (Sheehan et al, 1998), to exclude psychiatric, neurological, and medical history. Subjects were requested to complete questionnaires including the Beck Depression Inventory (Beck et al, 1961), Trait Anxiety Inventory (STAI) (Spielberger et al, 1970), and Barratt Impulsiveness Scale (BIS) (Patton et al, 1995). Symptoms of hyperactivity and attention deficits were assessed with the ADHD self-report screening questionnaire (DuPaul et al, 1998). Verbal Intelligence was assessed with the Dutch Adult Reading Test (NLV) (Schmand et al, 1991). A baseline measure of working memory capacity was assessed during intake with a Dutch version of the listening span (Daneman and Carpenter, 1980) and the digit span (Groth-Marnat, 2001).

\section{Pharmacological Design and Session Procedures}

A within-subject, double-blind, placebo-controlled crossover design was employed. Subjects were tested after administration of MPH (Ritalin, $20 \mathrm{mg}$ ) or placebo on two different occasions, separated by at least 1 week. All subjects abstained from alcohol or over-the-counter medication $24 \mathrm{~h}$ before testing and caffeine on the day of testing. They were asked to have a light breakfast one hour before arrival, similar across both sessions. The reversal learning task was assessed $\sim 165 \mathrm{~min}$ after drug intake for $\sim 20 \mathrm{~min}$, directly followed by the digit span. Ritalin is effective for $\sim 4 \mathrm{~h}$ with peak plasma levels $90 \mathrm{~min}$ after dosing (Swanson et al, 2003). Although time of testing was optimized for the preceding fMRI paradigm, assessment of the current task coincided with the active time window of drug effects. Physiology and mood were assessed $\sim 15$ min prior (T1), $\sim 30$ min after (T2) and $\sim 210 \mathrm{~min}$ (T3) after drug intake. See Supplementary Materials and Methods for analyses and results of physiology and mood.

\section{Baseline Working Memory Capacity}

Baseline working memory capacity was assessed with the digit span (Groth-Marnat, 2001) during intake and both 
drug sessions. As in our previous report (van der Schaaf et al, 2012), the average total digit span across all three assessments was selected for drug stratification, because it was thought to provide a more reliable estimate of working memory capacity owing to the fact that is was administered repeatedly. For each session, the individual total score on the digit span forward and digit span backward was calculated. These total scores were averaged across the three assessments and used as a covariate of interest in the behavioral analysis (see further below). In addition to the digit span, the listening span (Daneman and Carpenter, 1980) was assessed during intake (two missing values). To confirm our baseline-dependent results, supplementary analyses were done with listening span and the digit span assessed during intake (Supplementary Materials and Methods).

\section{Reversal Learning Paradigm}

We employed a reversal learning task used previously (Cools et al, 2006) (Figure 1). Two stimuli, a face and a scene, were presented simultaneously on the screen (location randomized). One of these stimuli was associated with reward and the other with punishment (or reward omission, note that we cannot disentangle the two). Subjects were required to learn these deterministic stimulus-outcome associations. Unlike standard (probabilistic) reversal paradigms, subjects did not choose between the two stimuli. Instead, one of the stimuli was already selected by the computer (highlighted with a black border) and subjects were asked to predict the outcome of this preselected stimulus. After the prediction, indicated with a right index or middle finger button press (counterbalanced between subjects), the actual outcome was presented after a 1000 -ms delay for $500 \mathrm{~ms}$ at the location of the stimulus. There was no time limit to provide a response. Reward consisted of a green smiley with a ' $+\$ 100$ ' sign. Punishment consisted of a red sad smiley and a ' $-\$ 100$ ' sign. Note that this outcome did not depend on subjects' responses but was directly coupled to the stimulus. After 4-6 consecutive correct predictions the stimulus-outcome contingency reversed. This was either signaled by an unexpected reward, presented after the previously punished stimulus was highlighted, or by an unexpected punishment, presented after the previously rewarded stimulus was highlighted. Accuracy on the trials directly following these unexpected outcomes (reversal trials) represents how well subjects updated their stimulusoutcome associations. After unexpected outcomes, the same stimulus was highlighted again, such that behavioral and cognitive requirements were matched between valence conditions.

Each participant performed four experimental blocks that contained 120 trials: two blocks in which reversals were signaled by unexpected rewards (reward condition) and two blocks in which reversals were signaled by unexpected punishment (punishment condition). Each block consisted of one acquisition stage until the first reversal and a variable number of reversal stages, depending on the participant's accuracy. On average, each participant performed 23.5 ( \pm 5.4$)$ and $23.3( \pm 4.7)$ reversal stages in the punishment and reward condition, respectively. On each session,
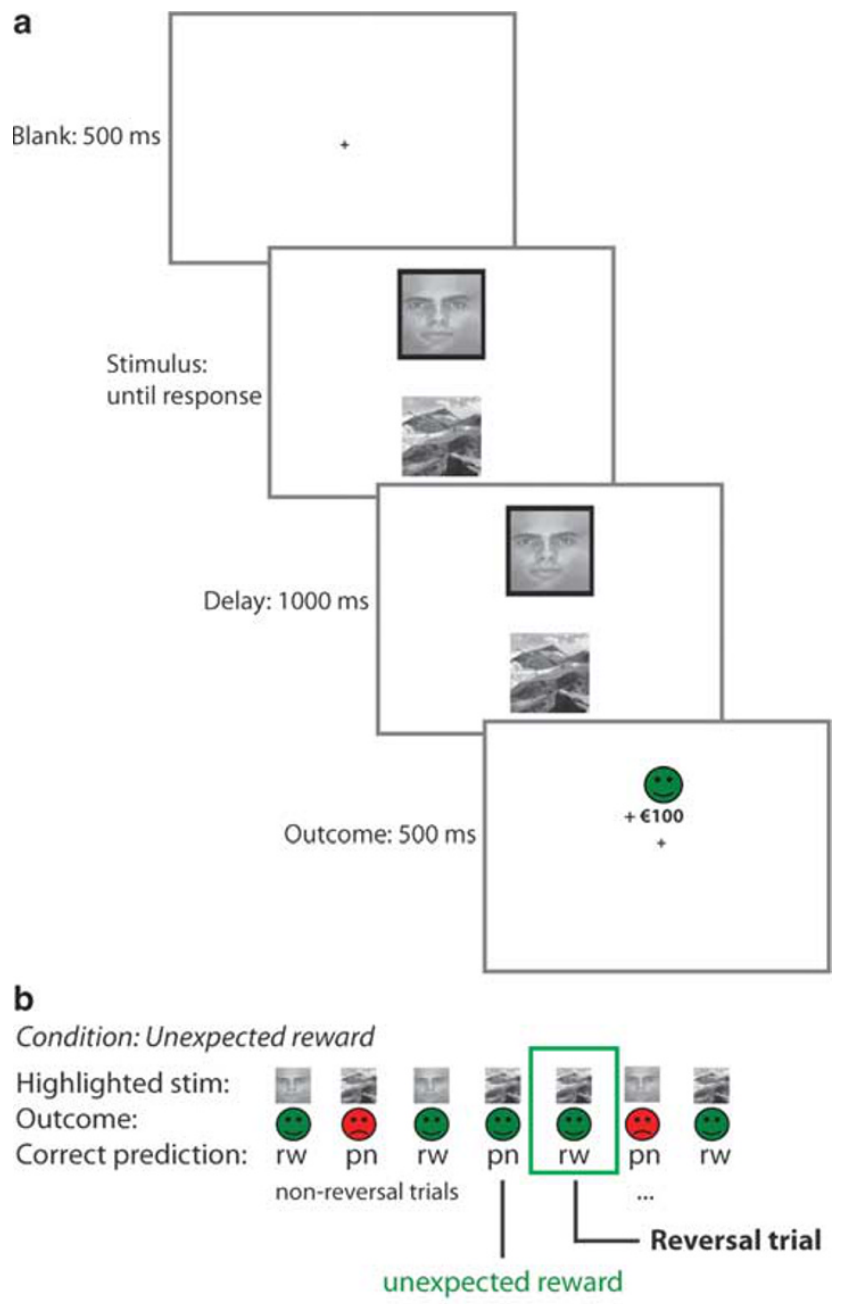

Condition: Unexpected punishment Highlighted stim: Outcome: Correct prediction:

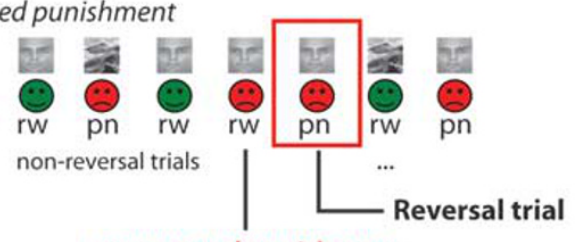

unexpected punishment

Figure I Task design. (a) Example of a reward trial. Two stimuli, a face and a scene, were presented simultaneously. One of the two stimuli was highlighted with a black border and the task was to predict whether this stimulus was followed by reward or punishment, after which the actual outcome was presented ( $100 \%$ deterministic). (b) Example of a trial sequence for the unexpected reward and unexpected punishment condition. The participant learned to predict rewards (rw) and punishments (pn) for the scene and face. Stimulus-outcome associations reversed after 4-6 consecutive correct predictions, signaled by either unexpected reward or unexpected punishment. Measure of interest was the accuracy on reversal trials immediately following the unexpected outcomes.

subjects performed two practice blocks to familiarize them with the paradigm. Performance in the experimental blocks was above chance level ( $>70 \%$ correct) for all subjects and there were no test-retest effects. See Supplementary Materials and Methods for test-retest effects and information about randomization and practice blocks. 


\section{Behavioral Data Analysis}

There were three trial-types per valence condition: reversal, non-reversal reward, and non-reversal punishment. Reversal trials were defined as those trials following an unexpected outcome. Non-reversal trials were defined as those trials following expected outcomes and preceding expected rewards (non-reversal reward) or expected punishments (non-reversal punishment). Only trials from the reversal stages (after the first unexpected outcome) and trials following correct predictions were included in the analysis.

Proportions of correct responses per trial-type were arcsine transformed $(2 x \operatorname{arcsine}(\sqrt{ } x))$ as is appropriate when the variance is proportional to the mean (Howell, 1997). To investigate whether effects of MPH on reversal learning depend on baseline working memory, we employed a repeated measures ANOVA with the within-subject factors: drug (placebo, MPH), valence (reward, punishment), and trial-type (reversal, non-reversal reward, nonreversal punishment); and baseline working memory capacity (mean centered) as covariate of interest. Greenhousse-Geiser correction was applied when sphericity assumption was violated. Linear relationships between valence-dependent learning and baseline working memory capacity were further assessed using Pearson correlation analysis. To this end, valence-dependent reversal learning scores were calculated by computing the difference between the proportion of correct responses on reward and punishment reversal trials. This measure was then correlated with individual measures of baseline working memory. We specifically focused our analyses on relative scores because this measure controls for nonspecific drug effects such as changes in effort, attention, or alertness. The only measure of interest that was not normally distributed was the relative (valence-dependent) reversal score in the MPH condition (Shapiro-Wilk test: $P=0.016$, uncorrected for multiple comparisons). Therefore we also report the nonparametric (Spearman's rho) correlation for our primary effect of interest.

\section{Supplementary Win-Stay Lose-Shift Analysis}

Both reward and punishment reversal trials required response alternation. Therefore, it could be argued that changes on valence-dependent learning reflect changes in the adoption of a win-stay/lose-shift strategy, ie, the tendency to maintain responding after reward (win-stay) and to alternate responses after punishment (lose-shift). Thus, improvements on reward relative to punishment learning could reflect a bias away from a win-stay/lose-shift strategy or a bias toward a win-shift/lose-stay strategy, rather than increased ability to update reward relative to punishment predictions. To test this alternative hypothesis, we measured the adoption of a win-stay/lose-shift strategy on the non-reversal trials. We calculated accuracy scores for the following four trial-types averaged across valence conditions: non-reversal reward trials after correctly predicted rewards (win-stay) and correctly predicted punishment trials (lose-shift), and non-reversal punishment trials after correctly predicted rewards (win-shift) and correctly predicted punishments (lose-stay). Drug effects were assessed with repeated measures ANOVA with the within-subject factors: drug (placebo, MPH), strategy (stay, shift), and outcome (reward, punishment); and working memory capacity as covariate. Direct associations between drug effects on valence-dependent reversal learning and strategy were assessed with correlation analyses. To this end, individual levels of a win-stay/lose-shift strategy was calculated as ((win-stay + lose-shift)-(win-shift + lose-stay)), where higher values reflect a greater adoption of a win-stay/lose-shift strategy.

\section{RESULTS}

\section{Subjects}

All 19 subjects were healthy and none met DSM-IV criteria for ADHD as measured with the self-report symptom questionnaire (symptoms of inattention child: $1.3 \pm 1.6$, range: $0-6$; adult: $0.6 \pm 0.8$, range: $0-3$; symptoms of hyperactivity child: $1.7 \pm 1.6$, range: $0-5$; adult: $1.3 \pm 1.3$, range: $0-5$ ) or depression as measured with the Beck Depression Inventory $(1.4 \pm 2.0$, range: $0-5)$. All had normal levels of trait anxiety (STAI: $30.1 \pm 5.5$, range: $22-42$ ) and impulsivity (BIS: $61.1 \pm 8.0$, range: 41-74). There were no associations between these baseline measures and MPH-induced changes on valence-dependent reversal learning (all $P>0.1$ ).

\section{Effects of MPH on Reward vs Punishment Learning Varied as a Function of Baseline Working Memory Capacity}

MPH was predicted to alter reward $v s$ punishment reversal learning as a function of baseline working memory capacity. This was confirmed statistically by a significant drug $\times$ valence $\times$ trial-type $\times$ span interaction $\quad\left(\mathrm{F}_{16,2}=12.8\right.$, $P<0.001)$. A breakdown of this interaction confirmed that the drug $\times$ valence $\times$ span interaction was significant for the reversal trials $\left(\mathrm{F}_{17,1}=23.77, P<0.001\right)$, but not for the nonreversal reward $\left(\mathrm{F}_{17,1}=0.02\right.$, ns) or non-reversal punishment trials $\left(F_{17,1}=2.36, P=0.2\right)$. Correlation analysis revealed a positive relationship between working memory capacity and MPH effects on valence-dependent reversal learning scores $\left(r_{19, \text { Pearson }}=0.76, P<0.001 ; r_{19, \text { Spearman's rho }}=0.72, P<0.001\right)$. Thus, we show that MPH improved reward $v s$ punishment learning in high-working memory subjects, while the opposite was seen in low-working memory subjects (Figure 2; Table 1; Supplementary Figure S1). All baseline-dependent effects were replicated with the digit span and listening span assessed during intake (Supplementary Materials and Methods). MPH did not affect digit span itself (drug $\times$ span: $F_{18,1}=0.11$, ns; main effect of drug: $\mathrm{F}_{18,1}=1.12, P=0.3$ ), also not as a function of digit span assessed during intake (drug $\times$ baseline $\times$ span: $\mathrm{F}_{18,1}=0.11$, ns) (Table 2).

Subjective mood and physiology effects were as predicted with higher reports of subjective alertness and positive affect as well as heart rate and systolic and diastolic blood pressure increases after administration of MPH relative to placebo across all subjects (Supplementary Table S1). These $\mathrm{MPH}$-induced changes on mood and physiology were not associated with MPH-induced changes on valence-dependent reversal learning (all $P>0.1$ ) (see Supplementary Materials and Methods for details). 


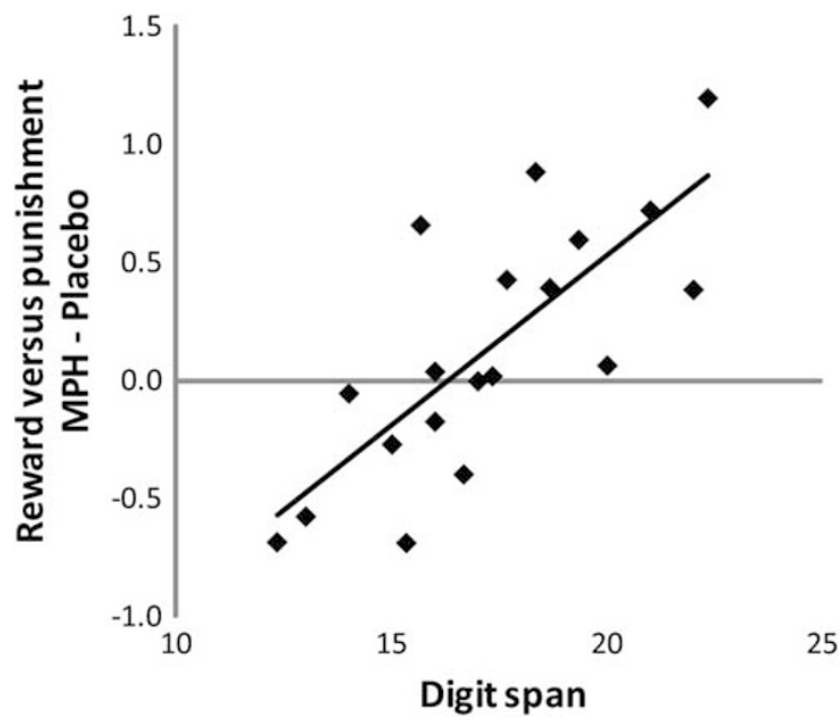

Figure 2 Linear relationship between baseline working memory capacity (digit span) and methylphenidate (MPH) effects on reward vs punishment learning $(r=0.69, P<0.00 I)$. Data on the $y$-axis reflect arcsine-transformed valence-dependent reversal scores (ie accuracy on reward relative to punishment reversal trials) after administration of MPH relative to placebo. Diamonds represent individual data points.

Table I Behavioral Data on the Reversal Learning Task

\begin{tabular}{|c|c|c|}
\hline & Placebo & MPH \\
\hline \multicolumn{3}{|l|}{ Reward condition } \\
\hline Reversal & $0.95(0.01)$ & $0.98(0.01)$ \\
\hline Non-reversal reward & $0.96(0.01)$ & $0.96(0.01)$ \\
\hline Non-reversal punishment & $0.95(0.01)$ & $0.97(0.01)$ \\
\hline \multicolumn{3}{|l|}{ Punishment condition } \\
\hline Reversal & $0.95(0.02)$ & $0.96(0.01)$ \\
\hline Non-reversal reward & $0.94(0.01)$ & $0.97(0.01)$ \\
\hline Non-reversal punishment & $0.94(0.01)$ & $0.96(0.01)$ \\
\hline
\end{tabular}

Abbreviation: $\mathrm{MPH}$, methylphenidate.

Values represent raw accuracy scores (SE) per trial-type and drug condition averaged across all subjects $(N=19)$. Reversal trials are presented in bold.

\section{Supplementary Win-Stay, Lose-Shift Analysis}

Effects on valence-dependent reversal learning could not be attributed to overall MPH-induced changes on the adoption of a win-stay/lose-shift strategy. Thus, analysis of MPH effects on win-stay/lose-shift strategy did not reveal any span-dependent effect. There was no drug $\times$ strategy $\times$ outcome $\times$ span interaction $\left(\mathrm{F}_{17,1}=0.004, \mathrm{~ns}\right)$. Furthermore, correlation analysis revealed that MPH-induced changes on valence-dependent reversal learning were not associated with MPH-induced changes on a win-stay/lose-shift strategy $\left(r_{19}=0.015, \mathrm{~ns}\right)$.

An additional analysis was conducted to assess whether there were MPH effects on response strategy irrespective of working memory capacity. This analysis revealed a significant drug $\times$ strategy $\times$ outcome interaction $\left(\mathrm{F}_{17}=6.77, P=0.019\right)$. Post-hoc pair-wise comparisons for
Table 2 Digit Span

\begin{tabular}{lcccr}
\hline & Intake & Placebo & MPH & Average \\
\hline Forward & $8.37(1.92)^{\mathrm{a}}$ & $9.32(2.31)$ & $9.79(1.93)$ & $9.16(1.62)$ \\
Backward & $7.58(1.54)^{\mathrm{a}}$ & $8.21(2.1)$ & $8.47(1.74)$ & $8.09(1.57)$ \\
Total & $15.95(2.9 \mid)^{\mathrm{ab}}$ & $17.53(3.73)$ & $18.26(3.14)$ & $17.25(2.84)$ \\
\hline
\end{tabular}

Abbreviation: $\mathrm{MPH}$, methylphenidate.

aDiffers significantly $(P<0.05)$ from the methylphenidate session.

biffers significantly $(P<0.05)$ from the placebo session.

Values represent forward, backward, and total span scores (SD) averaged across all subjects $(N=19)$ measured during intake, placebo, and methylphenidate session, and averaged across all measurements.

Table 3 Raw Accuracy Scores on Win-Stay, Lose-Stay, Win-Shift, and Lose-Shift Trials

\begin{tabular}{lll}
\hline & MPH & Placebo \\
\hline Win-stay & $0.86(0.03)$ & $0.86(0.03)$ \\
Lose-stay & $0.86(0.04)$ & $0.85(0.03)$ \\
Win-shift & $0.82(0.04)^{\mathrm{a}}$ & $0.85(0.04)$ \\
Lose-shift & $0.82(0.04)$ & $0.81(0.06)$ \\
\hline
\end{tabular}

Abbreviation: $\mathrm{MPH}$, methylphenidate.

${ }^{a}$ Differs significantly $(P<0.001)$ from the placebo session.

Values represent raw accuracy scores (SE) per trial-type and drug condition averaged across all subjects $(N=19)$.

each of the four trial-types separately revealed that MPH selectively decreased accuracy on win-shift trials $\left(\mathrm{T}_{18}=-4.17, P<0.001\right)$, while having no effect on winstay $\left(\mathrm{T}_{18}=0.053, P=0.95\right)$, lose-stay $\left(\mathrm{T}_{18}=0.25, P=0.8\right)$, and lose-shift trials $\left(\mathrm{T}_{18}=5, P=0.63\right)$ (Table 3 ).

\section{DISCUSSION}

The increased use of licensed stimulants like MPH by students in educational settings has raised questions about its cognitive-enhancing effects in the healthy population (Greely et al, 2008). One major issue is the large variability in the effects of such smart drugs on learning and cognition, both within and across individuals. Here we show that effects of MPH on reversal learning vary as function of baseline working memory capacity. Moreover, as predicted, effects of MPH were valence-dependent, so that MPH altered reward relative to punishment learning. Specifically, it improved reward $v s$ punishment learning in high-working memory subjects, while impairing it in low-working memory subjects. These effects could not be accounted for by nonspecific drug effects, for example on alertness or other subjective effects. These results elucidate two factors that contribute to the high variability of smart drug efficacy in the healthy population. First, they demonstrate that effects of MPH on learning vary within individuals as a function of the specific demands of the task, with differential effects on reward and punishment learning. Second, they demonstrate that effects of MPH on learning vary between individuals as a function of baseline working memory capacity, with opposite MPH effects in high- and 
low-working memory subjects. These results help understand the nature of the large inter- and intra-individual differences in the response to smart drugs like MPH.

Our results are generally consistent with previous work demonstrating opposite effects of dopaminergic drugs on reward and punishment learning in healthy subjects (Cools et al, 2009; Frank and O'Reilly, 2006; van der Schaaf et al, 2012) and further support computational modeling work, which suggests that striatal dopamine shifts the balance between reward and punishment learning (Frank, 2005; Maia and Frank, 2011). More specifically, we observed that $\mathrm{MPH}$ induced a larger improvement in reward $v s$ punishment learning in subjects with higher baseline working memory capacity. This observation is particularly relevant in the context of smart drug use in universities (Greely et al, 2008; Maher, 2008; Smith and Farah, 2011), given the large body of evidence supporting a substantial relationship between working memory capacity and general fluid intelligence (eg, Engle et al, 1999). Thus, general fluid intelligence might help predict whether smart drugs help or hurt. Baseline working memory capacity has been previously shown to be a putative proxy of baseline dopamine synthesis capacity in the striatum (Cools et al, 2008; Landau et al, 2009). Accordingly, the dependency on working memory capacity might reflect dependency on dopamine synthesis capacity, with larger improvements in reward $v s$ punishment learning in subjects with higher dopamine synthesis capacity. Our results are therefore consistent with the dopamine cell-activity hypothesis (Volkow et al, 2002), suggesting that DAT blockade induces larger dopamine increases, and thus larger improvements in reward $v s$ punishment learning (Frank, 2005; Maia and Frank, 2011), in subjects with high relative to low dopamine cell activity (Volkow et al, 2002). Furthermore, it is also in line, albeit indirectly, with prior work revealing larger MPH-induced impairments on punishment-based reversal learning in subjects with larger $\mathrm{MPH}$-induced increases in dopamine release (Clatworthy et al, 2009).

It might be noted that the present study revealed MPHinduced impairments in reward $v s$ punishment learning, presumably associated with decreases in dopamine (Frank, 2005), in subjects with low-working memory capacity. This aspect of our findings is not easily accounted for by the above-described cell-activity hypothesis, unless MPH acts more readily via autoregulatory (D2) systems in low- than in high-working memory/dopamine synthesis subjects. Further investigation is needed to elucidate the involvement of these additional mechanisms in the observed spandependent effects of MPH. Accordingly, we refrain here from definitively interpreting the MPH-induced impairments in mechanistic terms. Instead, we emphasize the relevance of the findings in the context of smart drug use by healthy adults, by suggesting that baseline working memory capacity may provide a valuable prediction measure for individual MPH effects.

At first sight, our findings might appear inconsistent with recent work showing beneficial effects of MPH on various cognitive tasks, in subjects who perform poorly at baseline (Eagle et al, 2007; Finke et al, 2010), consistent with the well-known inverted-U-shaped relationship between dopamine and cognitive performance (Arnsten, 1997). However, it should be noted that our study did in fact also reveal improvement in performance in low-span subjects, but only if performance is calculated in terms of punishment $v s$ reward learning. This illustrates one important take-home message of the present study, that effects of MPH improve or impair cognition depending on current task demands.

We did not find any MPH effect on working memory capacity itself, as measured with the digit span. This is consistent with our previous report (van der Schaaf et al, 2012) and various other reports (Oken et al, 1995; Schmedtje et al, 1988; Silber et al, 2006; but see Agay et al, 2010), but in apparent contrast with other studies reporting stimulant effects on more complex spatial working memory tasks (Clatworthy et al, 2009; Mehta et al, 2000). In addition, the direction of our (positive) association between digit span and MPH's effects on reward vs punishment learning is also in contrast with that observed previously for spatial working memory (Mehta et al, 2000). Thus, Mehta et al (2000) have reported a negative rather than a positive association between digit span and MPH's effects on spatial working memory. One explanation for this apparent discrepancy could be that the tasks have different cognitive requirements. Indeed, together with prior work by Clatworthy et al (2009), the current study indicates that the nature of the relationship between the cognitive effects of MPH and the baseline state of the system depends critically on task demands.

MPH sustained subjective feelings of alertness and positive affect over time and increased heart rate and blood pressure across all subjects. Importantly, these nonspecific effects of MPH could not explain the effects of interest on valence-dependent learning, because, unlike our effects of interest, they did not depend on working memory capacity. One possibility is that these effects reflect modulation of (prefrontal) noradrenalin, known to be involved in sympathetic control, attention, and executive functioning (Arnsten, 1997).

Effects on valence-dependent reversal learning could not be attributed to MPH-induced changes on the adoption of a win-stay/lose-shift strategy as measured on the non-reversal trials. Supplementary analysis did reveal that MPH selectively decreased the tendency to shift responding (on nonreversal punishment trials) after non-reversal reward trials, suggesting enhanced stickiness or response perseveration after reward. Although these results are potentially interesting, our task was not designed to directly assess $\mathrm{MPH}$ effects on instrumental response strategy. Accordingly, these results should be considered as preliminary, and future studies specifically designed to assess instrumental response strategy should further explore these potential effects of MPH. Stimulant medication has been shown to ameliorate reward $v s$ punishment learning deficits in patients with ADHD (Frank et al, 2007). ADHD has been associated with low (spatial) working memory (Barkley, 1997) and striatal dopamine deficiency (Volkow et al, 2009). Thus, the MPH-induced improvement in ADHD seems at odds with the present finding that MPH impaired reward relative to punishment learning in healthy adults with lowworking memory capacity. This discrepancy might reflect the fact that beneficial effects of $\mathrm{MPH}$ on reward learning have been shown only in ADHD patients who have received long-term stimulant treatment. Long-term stimulant 
treatment might have effects that are quite different from those of acute administration (Robbins, 2002). Indeed longterm stimulant treatment might induce changes in DAT expression (Fusar-Poli et al, 2012) and/or dopamine signaling (Grace, 1991; Volkow et al, 2012). Accordingly, the current study of acute MPH administration in the healthy population provides a better model of MPH use in healthy adults, who likely take smart pills only on particular occasions (Smith and Farah, 2011) and not on the longer term.

The finding that MPH has differential effects on reward and punishment learning might have relevance to other task domains, given that many tasks load on either reward or punishment learning. Thus, improved reward (relative to punishment) learning might well translate to enhanced attribution of positive value to study material and thus increase student interest and motivation in schoolwork. In line with that, mesolimbic dopamine is thought to modulate the attribution of incentive salience to stimuli that drive behavior and become the focus of goal-directed behavior (Berridge and Robinson, 1998). This is further supported by a positive correlation between $\mathrm{MPH}$-induced extracellular dopamine increases and subjective ratings of interest, excitement, and motivation for a mathematical task (Volkow et al, 2004). Conversely, improved punishment (relative to reward) learning might translate to enhanced attribution of negative value, something characteristic of mood disorders like depression (Clark et al, 2009; Robinson et al, 2011). This also raises concerns about possible negative side effects of MPH and highlights the need for further evaluation of both potential risks and benefits of cognitive enhancement in the healthy population. Moreover, future studies are needed to further unravel how changes in reward and punishment learning might contribute to academic performance.

\section{ACKNOWLEDGEMENTS}

$\mathrm{RC}$ is supported by a VIDI grant from the innovational Incentives Scheme of the Netherlands Organization for Scientific Research (NWO), a research grant to Kae Nakamura, RC, and Nathaniel Daw from the Human Frontiers Science Program (RGP0036/2009-C), and the 2012 James McDonnell Scholar Award.

\section{DISCLOSURE}

$\mathrm{RC}$ has been a consultant to Abbott Laboratories, but she is not an employee or a stock shareholder. JB has been in the past 3 years a consultant to/ member of advisory board of/ and speaker for Janssen Cilag BV, Eli Lilly, Bristol-Myer Squibb, Shering Plough, UCB, Shire, Novartis and Servier, but he is not an employee or a stock shareholder of any of these companies. He has no other financial or material support, including expert testimony, patents or royalties.

\section{DISCLAIMER}

All listed authors concur in submission and the final manuscript has been approved by all. Experimental procedures have been conducted in conformance with the policies and principles contained in the Federal Policy for the Protection of Human Subjects and in the Declaration of Helsinki. The paper has not been and is not intended to be published anywhere in any language except in Neuropsychopharmacology. No similar paper has been, or will be, simultaneously submitted for publication elsewhere.

\section{REFERENCES}

Agay N, Yechiam E, Carmel Z, Levkovitz Y (2010). Non-specific effects of methylphenidate (Ritalin) on cognitive ability and decision-making of ADHD and healthy adults. Psychopharmacology 210: 511-519.

Arnsten A (1997). Catecholamine regulation of the prefrontal cortex. J Psychopharmacol 11: 151-162.

Barkley R (1997). Behavioral inhibition, sustained attention, and executive functions: constructing a unifying theory of ADHD. Psychol Bull 121: 65-159.

Beck A, Ward C, Mendelson M, Mock J, Erbaugh J (1961). An inventory for measuring depression. Arch Gen Psychiatry 4: 561-632.

Berridge K, Robinson T (1998). What is the role of dopamine in reward: hedonic impact, reward learning, or incentive salience? Brain Res Brain Res Rev 28: 309-369.

Bodi N, Keri S, Nagy H, Moustafa A, Myers CE, Daw N et al (2009). Reward-learning and the novelty-seeking personality: a betweenand within-subjects study of the effects of dopamine agonists on young Parkinson's patients. Brain 132(Pt 9): 2385-2395.

Bundt C, van Mil A, Olthof B, Reintjes W (2012). Presteren onder druk of drugs? Een interdisciplinaire academische verkenning naar cognitive enhancement. In: Radboud Honours Academy (eds) The Wider Implications of Cognitive Neuroscience, Reflections on Science 2011-2012. Radboud Honours Academy: Nijmegen.

Clark L, Chamberlain S, Sahakian B (2009). Neurocognitive mechanisms in depression: implications for treatment. Annu Rev Neurosci 32: 57-74.

Clatworthy P, Lewis S, Brichard L, Hong Y, Izquierdo D, Clark L et al (2009). Dopamine release in dissociable striatal subregions predicts the different effects of oral methylphenidate on reversal learning and spatial working memory. J Neurosci 29: 4690-4696.

Cools R, Altamirano L, D’Esposito M (2006). Reversal learning in Parkinson's disease depends on medication status and outcome valence. Neuropsychologia 44: 1663-1673.

Cools R, D'Esposito M (2011). Inverted-u-shaped dopamine actions on human working memory and cognitive control. Biol Psychiatry 69: e113-e125.

Cools R, Frank MJ, Gibbs SE, Miyakawa A, Jagust W, D’Esposito M (2009). Striatal dopamine predicts outcome-specific reversal learning and its sensitivity to dopaminergic drug administration. J Neurosci 29: 1538-1543.

Cools R, Gibbs SE, Miyakawa A, Jagust W, D’Esposito M (2008). Working memory capacity predicts dopamine synthesis capacity in the human striatum. J Neurosci 28: 1208-1212.

Daneman M, Carpenter PA (1980). Individual differences in working memory and reading. J Verb Learn Verb Beh 19: 450-916.

DuPaul G, Power T, Anastopoulos A, Reid R (1998). ADHD Rating Scale - IV: Checklists, Norms, and Clinical Interpretation.

Eagle D, Tufft M, Goodchild H, Robbins T (2007). Differential effects of modafinil and methylphenidate on stop-signal reaction time task performance in the rat, and interactions with the dopamine receptor antagonist cis-flupenthixol. Psychopharmacology 192: 193-206.

Engle R, Tuholski S, Laughlin J, Conway A (1999). Working memory, short-term memory, and general fluid intelligence: a latent-variable approach. J Exp Psychol Gen 128: 309-331. 
Faraone S, Buitelaar J (2010). Comparing the efficacy of stimulants for ADHD in children and adolescents using meta-analysis. Eur Child Adolesc Psychiatry 19: 353-417.

Finke K, Dodds C, Bublak P, Regenthal R, Baumann F, Manly T et al (2010). Effects of modafinil and methylphenidate on visual attention capacity: a TVA-based study. Psychopharmacology 210: 317-329.

Frank M, O'Reilly R (2006). A mechanistic account of striatal dopamine function in human cognition: psychopharmacological studies with cabergoline and haloperidol. Behav Neurosci 120: 497-1014.

Frank MJ (2005). Dynamic dopamine modulation in the basal ganglia: a neurocomputational account of cognitive deficits in medicated and nonmedicated Parkinsonism. J Cogn Neurosci 17: 51-72.

Frank MJ, Santamaria A, O'Reilly RC, Willcutt E (2007). Testing computational models of dopamine and noradrenaline dysfunction in attention deficit/hyperactivity disorder. Neuropsychopharmacology 32: 1583-1599.

Fusar-Poli P, Rubia K, Rossi G, Sartori G, Balottin U (2012). Striatal dopamine transporter alterations in ADHD: pathophysiology or adaptation to psychostimulants? A meta-analysis. Am J Psychiatry 169: 264-272.

Grace A (1991). Phasic versus tonic dopamine release and the modulation of dopamine system responsivity: a hypothesis for the etiology of schizophrenia. Neuroscience 41: 1-25.

Greely H, Sahakian B, Harris J, Kessler R, Gazzaniga M, Campbell P et al (2008). Towards responsible use of cognitive-enhancing drugs by the healthy. Nature 456: 702-705.

Groth-Marnat G (ed) (2001). The Wechsler Intelligence Scales. Cambridge University Press: Cambridge.

Hooks M, Colvin A, Juncos J, Justice J (1992). Individual differences in basal and cocaine-stimulated extracellular dopamine in the nucleus accumbens using quantitative microdialysis. Brain Res 587: 306-318.

Howell DC (1997). Statistical Methods for Psychology. Wadsworth Publishing Company.

Kimberg DY, D’Esposito M, Farah MJ (1997). Effects of bromocriptine on human subjects depend on working memory capacity. Neuroreport 8: 3581-3585.

Landau SM, Lal R, O’Neil JP, Baker S, Jagust WJ (2009). Striatal dopamine and working memory. Cereb Cortex 19: 445-454.

Maher B (2008). Poll results: look who's doping. Nature 452: 674-675.

Maia TV, Frank MJ (2011). From reinforcement learning models to psychiatric and neurological disorders. Nat Neurosci 14: 154-162.

Marcus S, Durkin M (2011). Stimulant adherence and academic performance in urban youth with attention-deficit/hyperactivity disorder. J Am Acad Child Adolesc Psychiatry 50: 480-489.

Mehta M, Goodyer I, Sahakian B (2004). Methylphenidate improves working memory and set-shifting in $\mathrm{AD} / \mathrm{HD}$ : relationships to baseline memory capacity. J Child Psychol Psychiatry 45: 293-598.

Mehta M, Owen A, Sahakian B, Mavaddat N, Pickard J, Robbins T (2000). Methylphenidate enhances working memory by modulating discrete frontal and parietal lobe regions in the human brain. J Neurosci 20: 6 .

Mehta MA, Swainson R, Ogilvie AD, Sahakian J, Robbins TW (2001). Improved short-term spatial memory but impaired reversal learning following the dopamine $\mathrm{D}(2)$ agonist bromocriptine in human volunteers. Psychopharmacology 159: 10-20.

Oken B, Kishiyama S, Salinsky M (1995). Pharmacologically induced changes in arousal: effects on behavioral and electrophysiologic measures of alertness and attention. Electroencephalogr Clin Neurophysiol 95: 359-371.
Patton J, Stanford M, Barratt E (1995). Factor structure of the Barratt impulsiveness scale. J Clin Psychol 51: 768-842.

Robbins T (2002). ADHD and addiction. Nat Med 8: 24-29.

Robinson O, Cools R, Carlisi C, Sahakian B, Drevets W (2011). Ventral striatum response during reward and punishment reversal learning in unmedicated major depressive disorder. Am J Psychiatry 169: 152-159.

Schmand B, Bakker D, Saan R, Louman J (1991). The Dutch Reading Test for Adults: a measure of premorbid intelligence level. Tijdschr Gerontol Geriatr 22: 15-24.

Schmedtje J, Oman C, Letz R, Baker E (1988). Effects of scopolamine and dextroamphetamine on human performance. Aviat Space Environ Med 59: 407-410.

Sheehan D, Lecrubier Y, Sheehan K, Amorim P, Janavs J, Weiller E et al (1998). The Mini-International Neuropsychiatric Interview (M.I.N.I.): the development and validation of a structured diagnostic psychiatric interview for DSM-IV and ICD-10. J Clin Psychiatry 59(Suppl 20): 22-33.

Silber B, Croft R, Papafotiou K, Stough C (2006). The acute effects of d-amphetamine and methamphetamine on attention and psychomotor performance. Psychopharmacology 187: 154-169.

Smith M, Farah M (2011). Are prescription stimulants "smart pills"? The epidemiology and cognitive neuroscience of prescription stimulant use by normal healthy individuals. Psychol Bull 137: 717-741.

Spielberger G, Gorsuch RL, Lushene RE (1970). Manual for the State-Trait Anxiety Inventory. Consulting Psychologists Press: Palo Alto, CA, USA.

Swanson J, Gupta S, Lam A, Shoulson I, Lerner M, Modi N et al (2003). Development of a new once-a-day formulation of methylphenidate for the treatment of attention-deficit/hyperactivity disorder: proof-of-concept and proof-of-product studies. Arch Gen Psychiatry 60: 204-215.

van der Schaaf M, van Schouwenburg M, Geurts D, Schellekens A, Buitelaar J, Verkes R et al (2012). Establishing the dopamine dependency of human striatal signals during reward and punishment reversal learning. Cereb Cortex (e-pub ahead of print, doi:10.1093/cercor/bhs344).

Volkow N, Wang G-J, Fowler J, Logan J, Franceschi D, Maynard L et al (2002). Relationship between blockade of dopamine transporters by oral methylphenidate and the increases in extracellular dopamine: therapeutic implications. Synapse 43: 181-188.

Volkow N, Wang G-J, Fowler J, Telang F, Maynard L, Logan J et al (2004). Evidence that methylphenidate enhances the saliency of a mathematical task by increasing dopamine in the human brain. Am J Psychiatry 161: 1173-1180.

Volkow N, Wang G-J, Kollins S, Wigal T, Newcorn J, Telang F et al (2009). Evaluating dopamine reward pathway in ADHD: clinical implications. JAMA 302: 1084-1091.

Volkow N, Wang G-J, Tomasi D, Kollins S, Wigal T, Newcorn J et al (2012). Methylphenidate-elicited dopamine increases in ventral striatum are associated with long-term symptom improvement in adults with attention deficit hyperactivity disorder. J Neurosci 32: 841-850.

Voon V, Pessiglione M, Brezing C, Gallea C, Fernandez HH, Dolan RJ et al (2010). Mechanisms underlying dopaminemediated reward bias in compulsive behaviors. Neuron 65: 135-142.

Wigal S, Wigal T, Schuck S, Brams M, Williamson D, Armstrong R et al (2011). Academic, behavioral, and cognitive effects of OROS $($ methylphenidate on older children with attentiondeficit/hyperactivity disorder. J Child Adolesc Psychopharmacol 21: 121-131.

Supplementary Information accompanies the paper on the Neuropsychopharmacology website (http://www.nature.com/npp) 